\title{
Corela
}

Cognition, représentation, langage

18-1 | 2020

Vol. $18, \mathrm{n}^{\circ} 1$

\section{L'intégration morphologique des emprunts créoles dans la langue mancagne}

\section{Dame Ndao}

\section{(2) OpenEdition}

\section{Journals}

Édition électronique

URL : http://journals.openedition.org/corela/11367

DOI : 10.4000/corela. 11367

ISSN : 1638-573X

Éditeur

Cercle linguistique du Centre et de l'Ouest - CerLICO

\section{Référence électronique}

Dame Ndao, «L'intégration morphologique des emprunts créoles dans la langue mancagne », Corela [En ligne], 18-1 | 2020, mis en ligne le 26 juin 2020, consulté le 29 juin 2020. URL : http:// journals.openedition.org/corela/11367; DOI : https://doi.org/10.4000/corela.11367

Ce document a été généré automatiquement le 29 juin 2020.

\section{(c) (i) (2)(2)}

Corela - cognition, représentation, langage est mis à disposition selon les termes de la licence Creative Commons Attribution - Pas d'Utilisation Commerciale - Partage dans les Mêmes Conditions 4.0 International. 


\title{
L'intégration morphologique des emprunts créoles dans la langue mancagne
}

\author{
Dame Ndao
}

\section{Introduction}

1 L'une des particularités des langues atlantiques est que l'ensemble de leur lexique est réparti en classes nominales qui gouvernent un système d'accord. Dans le lexique mancagne, il existe beaucoup d'emprunts au créole de Bissau qui n'est pas une langue à classes $^{1}$. Cette situation, assez complexe n'a pas encore été l'objet d'étude. Toutefois, nous disposons de travaux scientifiques sur le créole bisséen et quelques rares travaux sur le mancagne, surtout en ce qui concerne la lexicologie.

2 Lorsqu'on examine attentivement le lexique, on constate que l'intégration de ces emprunts ne se fait pas de manière libre ; elle obéit à des règles différentes, contrairement à la quasi-totalité des langues du Sénégal où l'intégration est facilement prédictible. Par exemple en karon les emprunts du créole de Guinée-Bissau sont tous dépourvus de classes, en Bayot, c'est la classe e- qui est utilisée.

Dans cette réflexion, nous verrons quelles sont les différentes stratégies utilisées par la langue mancagne pour l'intégration des emprunts au créole de Bissau dans le système de classes nominales.

\subsection{Cadre théorique et méthodologique}

4 Plusieurs missions de terrains effectuées entre 2016 et 2018 à Ziguinchor et en GuinéeBissau nous ont permis de collecter un corpus de plus de 150 emprunts lexicaux du mancagne au créole de Bissau. Il existe quelques rares ouvrages lexicographiques consacrés à la langue mancagne comme Gaved \& Stammers. (2004). Le corpus constitué auprès des informateurs qui sont des locuteurs natifs mancagne nous a permis 
d'augmenter sensiblement les données obtenues à partir des travaux existants datant de très longtemps. En guise d'exemple, le travail de recherches de référence du mancagne est de Trifkovic (1969). Durant nos différentes missions de terrains en Guinée-Bissau et à Ziguinchor, les discussions, les réunions, les émissions de radio, nous servaient pour relever tout mot prononcé par un locuteur qui nous semblait être un emprunt au créole. Cette liste a été soumise à l'appréciation de chercheurs ${ }^{2}$ créolistes du LLACAN $^{3}$ et à des personnes ${ }^{4}$ bilingues (mancagne-créole)

5 L'approche des emprunts envisagée dans cette étude s'appuie sur la théorie de l'emprunt intégral tel que pensée par Loubier (2011) qui prend en compte l'emprunt intégral, hybride, le faux emprunt et le calque.

\subsection{Présentation du travail}

Dans ce travail nous nous proposons de présenter à travers des exemples précis les différentes formes qui marquent la complexité de l'intégration des emprunts du mancagne au créole de Guinée-Bissau. L'intégration est selon Demba (2000: 133), « l'acceptation (d'une) unité ou (d'un) trait linguistique étranger dans le moule de la langue emprunteuse » ou encore le processus d'acceptation d'unités étrangères dans le moule de la langue emprunteuse selon l'acception de Sambiéni (2012).

7 Dans un premier temps, nous présenterons l'ensemble du matériel morphologique lié à la classification nominale de ces langues. Dans un second temps, nous traiterons des emprunts par le système de classification nominale. En réalité, dans les langues atlantiques en général, et le mancagne en particulier, les emprunts sont toujours munis d'un affixe de classe dans leur intégration. Lorsque la première syllabe du mot emprunté est semblable à un préfixe, celle-ci fait office de marque de classe et alterne régulièrement avec la classe correspondante du pluriel comme par exemple : kaderno « cahier» / i-kaderno « cahiers ». Dans cette étude, nous essaierons de voir s'il existe des cas où la syllabe initiale identique à un préfixe de classe est remplacée par un authentique préfixe. Nous traiterons également des cas où la syllabe initiale ne correspond à aucune classe tout en mettant l'accent sur les motivations du choix des classes. Enfin, nous verrons si les appariements par défaut ainsi créés présentent une unité sémantique.

\section{Généralités}

8 Le système phonologique du mancagne présente selon Trifkovic (1969) 37 consonnes et 17 voyelles. C'est l'une des rares langues du Sénégal où la voyelle n'est pas soumise à l'harmonie vocalique et pourtant la langue possède un système ATR $^{5}$. Les radicaux monosyllabiques sont de structure CV « ka (richesse)», VC « ul (donner) », CVC « bob (termite) », CVCC « krank (paupière »). Dans les systèmes de classes nominales, tous les dépendants du nom sont soumis à l'accord de classe. Comme les autres langues du groupe $\mathrm{Bak}^{6}$ dont il fait partie, le mancagne marque les classes par des préfixes. Les classes sont définies par leur schème d'accord. L'accord concerne les démonstratifs, les adjectifs, les numéraux, les pronoms, les interrogatifs, etc.

\footnotetext{
1. ka toh $\mathrm{k}-\mathrm{i}$

CL maison CL-DEM

«Cette maison-ci »
} 


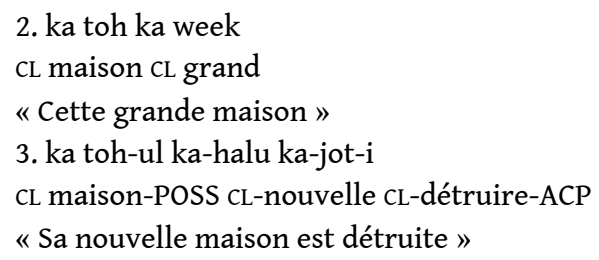

Les accords sont repartis, non pas entre deux genres comme en créole de Bissau, mais entre classes nominales marquées par un préfixe distinctif. Les classes sont appariées pour chaque nom, une pour le singulier, une pour le pluriel mais tous les noms d'une même classe au singulier n'ont pas forcément leur pluriel dans la même classe.

Sur le plan syntaxique, l'ordre très strict des constituants est SVO (Sujet-Verbe-Objet). On relève dans cette langue une hiérarchisation des pronoms ${ }^{7}$. Tous les arguments peuvent être focalisés et relativisés. Dans cette langue la relativisation d'un circonstant entraîne la disparition de la préposition, le verbe s'adjoint le suffixe un, ce qui est intéressant typologiquement ${ }^{8}$.

On classe habituellement, et à juste titre, le créole bisséen dans le groupe des créoles à base lexicale portugaise. De fait, c'est une langue qui est dépourvue de classes nominales.

A partir des exemples relevés, nous essaierons de mieux comprendre comment l'intégration des mots créoles empruntés se fait dans le système des classes nominales de la langue mancagne.

\section{Les préfixes nominaux}

13 Ils présentent 16 formes différentes qui sont de structure V- ou CV. Les différents préfixes nominaux sont munis d'un élément postposé devant les dépendants du nom présentant un accord de classe (cf ex. 1). Tous les éléments de l'énoncé sont munis d'une marque dont la forme dépend de la classe du nom. L'ensemble des formes ainsi définies constitue un schème d'accord.

- na a na-do 'créateur', a-nin 'mère'

- b b-duh 'menton'

- u u-pula 'serpent'

- ba ba-iin 'époux'

- m - m-neem 'oeuf'

- ka kə ka-hol 'épine'

- i i-tal 'cordes'

- pə pa pə-diim 'voix', pa-nel 'anneau'

- y- y-gut 'guerres'

- tə tə-ko 'endroit ici'

- də də-ko 'endroit là'

- ba bə ba-luk 'récompense' ba-duh 'menton'

\section{L'emprunt lexical}

Dès l'introduction Loubier (2011:5) attire notre attention sur le fait que les langues ne puissent pas se suffire à elles-mêmes et que le phénomène d'emprunt, source d'enrichissement, connait de nos jours un essor fulgurant. Pour paraphraser Kra (2016: 195), il existe plusieurs procédés de création lexicale qui sont utilisés dans toutes les 
langues du monde pour satisfaire aux besoins de communication. Parmi ces procédés nous pouvons citer la dérivation, la composition, le figement de syntagme, la réduplication, l'emprunt, etc. En raison de leur utilité, comme le souligne Builes (1998: 270), «...de nos jours plus encore qu'autrefois, les locuteurs ont besoin de créer de nouvelles unités significatives qui nomment les évolutions, voire les mutations techniques et sociales », les termes issus de ces procédés sont de plus en plus nombreux dans le stock lexical. Ils le sont davantage avec les emprunts. Au demeurant, il nous semble pertinent de souligner que la langue mancagne et le créole appartiennent à deux groupes linguistiques bien distincts. De ce fait, tout lexème commun est forcément un emprunt car la ressemblance ne peut être attribuée à une origine commune. Evoquer les emprunts, qui sont des faits de langue, c'est soulever de multiples problèmes tels que leur intégration dans la langue, les contacts historiques entre des communautés de locuteurs. L'emprunt résulte surtout de la notion de création lexicale, plus précisément d'un enrichissement lexical.

Il y a emprunt linguistique quand un parler A utilise et finit par intégrer une unité ou un trait linguistique qui existait précédemment dans un parler B et que A ne possède pas ; l'unité ou le trait emprunté sont eux-mêmes appelés emprunts (Dubois et al. 1973 : 188).

Cette définition vient préciser celle de Labatut (1983 : 41) selon laquelle, pour identifier un emprunt, il faut comparer deux lexèmes de deux langues différentes : ainsi tout lexème commun est nécessairement un emprunt.

Nous avons dans notre corpus 10 domaines sémantiques qui paraissent se prêter aux emprunts. Le tableau suivant est relatif aux thèmes de chaque domaine sémantique, à leur teneur en emprunts courants ou savants.

\begin{tabular}{|l|l|}
\hline Domaines & Thèmes \\
\hline Technique & nouveautés technologiques (machine, informatique, transport) \\
\hline Santé & Outils de travail, médicaments \\
\hline Militaire & nom d'agents, activités \\
\hline Religion & titres, activités \\
\hline Social & métiers, habillement nouveau, sports, médias \\
\hline Finance & moyens modernes d'épargne \\
\hline Agriculture & techniques, noms d'aliments étranger \\
\hline Justice & noms d'agents, activités et lieux \\
\hline Construction & Barrage, pont, nouvelles formes de constructions \\
\hline Education & matériel scolaire \\
\hline
\end{tabular}

L'examen du tableau ci-dessus montre que les emprunts savants dépassent de loin les emprunts courants. La classification des emprunts en thèmes révèle que c'est surtout 
dans le domaine de la technologie que les emprunts lexicaux sont nombreux. Il s'agit là de noms le plus souvent inconnus de la langue mancagne, soit parce que le concept est nouveau, soit parce qu'il y a abandon d'un terme autochtone au profit de l'emprunt.

\section{Les mécanismes d'intégration}

En s'inspirant de la manière dont Loubier (2011) juge l'acceptabilité et l'intégration des emprunts, nous nous appuyons sur les principes et les critères dégagés. Toujours selon Loubier (2011: 14) «L'emprunt lexical porte essentiellement sur le mot, dans sa relation sens-forme. Cette caractéristique le différencie des autres catégories, particulièrement de l'emprunt syntaxique et de l'emprunt phonétique ». C'est dans le lexique d'une langue que les emprunts sont les plus nombreux.

Dans notre corpus, nous avons dégagé quatre grands mécanismes d'intégration des emprunts créoles en macagne. Etant donné que les pratiques langagières sont réglées par les normes propres à chaque système de langue, nous allons dans les lignes suivantes, avec des exemples, préciser et analyser ces phénomènes d'intégration pour plus d'éclaircissements.

\subsection{Première syllabe semblable à un préfixe}

Si le mot comporte à l'initiale une séquence susceptible d'être interprétée comme un préfixe de classe, il sera affecté à la classe correspondante. Il faut signaler que c'est toujours le singulier qui entraîne le choix de la classe d'accueil. Le pluriel est alors formé de manière régulière, par commutation de l'élément interprété comme préfixe de singulier avec le préfixe de pluriel qui lui est le plus fréquemment associé. Ce procédé d'intégration concerne beaucoup de noms à initiale ka-, mais se rencontre dans la plupart des autres classes. Dans les tableaux ci-dessous nous avons aussi essayé de reprendre la filiation de l'emprunt jusqu'au portugais.

Initial ka-

\begin{tabular}{|l|l|l|l|}
\hline Portugais & Créole & Mancagne & Signification en français \\
\hline caderno & kadernu & Kaderno & « cahier » \\
\hline cassete & kaseti & kaseti & « cassette » \\
\hline camisa & kamisa & kamişa & « chemise » \\
\hline caro & karu & karu & « cher » \\
\hline cadeira & kadéra & karira & « chaise " \\
\hline
\end{tabular}

Initial p-

\begin{tabular}{|l|l|l|l|}
\hline Portugais & Créole & Mancagne & Signification en français \\
\hline
\end{tabular}




\begin{tabular}{|l|l|l|l|}
\hline prato & pratu & pratu & "plat » \\
\hline apitar & pitar & pitar & « siffler» \\
\hline pecadu & pekadu & pekadu & «péché » \\
\hline pedir & pidir & pidir & «prier » \\
\hline
\end{tabular}

Initial m-

\begin{tabular}{|l|l|l|l|}
\hline Portugais & Créole & Mancagne & Signification en français \\
\hline missa & misa & misa & « messe »; \\
\hline moru & muru & muurum & « maure » \\
\hline meza & mesa & meesa & « table » \\
\hline
\end{tabular}

Initial b-

\begin{tabular}{|l|l|l|l|}
\hline Portugais & Créole & Mancagne & Signification en français \\
\hline boneka & bonékar & boneka & « poupée » \\
\hline vela & bela & beela & « bougie »; \\
\hline bulir & bul-bulir & buliir & « remuer » \\
\hline
\end{tabular}

Cette syllabe qui fait office de marque de classe ne va pas alterner régulièrement avec la classe correspondante au pluriel contrairement à beaucoup de langues comme bijogo où on a kaasa "caisse " (pl. yaasa) ou en peul où on a lekkol «école " ngol. En mancagne, la norme est d'ajouter un préfixe de classe généralement i- et rarement $\mathrm{y}$ -

kaderno « cahier » (pl. i-kaderno)

\subsection{La syllabe initiale identique à un préfixe de classe mais remplacée}

Lorsque la forme du mot emprunté ne permet pas de lui attribuer "naturellement" une classe, le mot est affecté aux classes u- ou p- (singulier) et i (pluriel). Il est important de signaler que l'intégration des emprunts dans les classes en mancagne montre que statistiquement deux classes s'accaparent de la totalité des mots. Il s'agit de la classe $p$ qui est la classe fourre-tout et la classe $u$. A ma connaissance, il n'y a pas de motivation sémantique mais ces deux classes sont de loin les plus représentatives et sont très variées donc non marquées.

p-kaaju «pomme cajou»; p-kuraar « soigner »; p-kumsar « entamer »; p-kuuku « noix de coco »; u-pintura " peinture »; u-maakina, u-maakna « machine » u-mutur « moteur » 


\subsection{La syllabe initiale ne correspond à aucune classe}

Il existe en mancagne beaucoup de mots d'emprunts dont la syllabe initiale ne correspond à aucun préfixe de classe de la langue. Dans ce cas de figure notre corpus montre que la quasi-totalité de ces emprunts s'intègrent dans la classe $u$ -

u-mbarku « bateau », u-skol « école », u-gasolina « essence », u-feeru « marché »

\subsection{Les emprunts qui ne sont pas intégrés morphologiquement}

1 Il existe quelques rares mots empruntés qui ne prennent pas de marqueur de classe au singulier mais au pluriel la classe i-. L'explication que nous avons trouvée jusqu'ici est que ces mots, dans la conscience du locuteur mancagne, renvoient à un pluriel ou à un collectif.

\section{(rosadi « chapelet », batismo « baptême »)}

De plus, nous avons relevé quelques rares emprunts qui n'ont de marqueur de classe ni au singulier ni au pluriel. Des langues du sud du Sénégal comme le diola, il a été établi que l'absence ${ }^{9}$ de classes de certains emprunts au créole de Guinée-Bassau s'explique par le fait que ce sont d'anciens emprunts. Certes, l'hypothèse d'anciens emprunts peut être valable ici, mais même si les arguments solides font défaut, nous pensons également que le principe sémantique peut probablement jouer un rôle. Nous pensons que ces noms sont liés à des distinctions d'entités plus ou moins abstraites ou à des types de professions. Malheureusement en l'état actuel de nos recherches nous n'avons pas d'arguments précis, mais nous pensons qu'un traitement rigoureux avec des données plus exhaustives permettra d'avoir plus d'éclaircissements sur la question. Les exemples ci-dessous nous donnent une petite idée des motivations sémantiques.

manoobra « manœuvre », baluur « valeur », kanaaja « canaille »

Avant de terminer cette partie, nous avons jugé opportun de montrer qu'en mancagne on note une homophonie dans les schèmes d'accord avec le préfixe de classe à l'exception de na qui fait son accord en $a$.

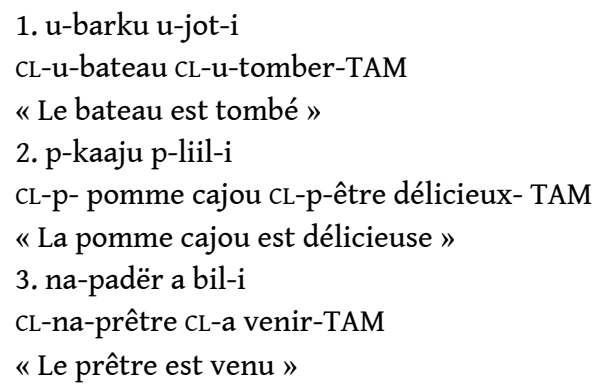

On voit bien que les emprunts qui ne sont pas intégrés morphologiquement dans le système des préfixes de classes cités un peu au-dessus, quand nous les mettons dans un schème d'accord, nous retrouvons la classe d'accord comme le montre l'exemple (4).

4. manoobra ki bil-i k- forsa

manœuvre CL-k-DET venir-TAM CL-k- fort

«Le manœuvre qui est venu est fort » 


\section{Synthèse sur l'intégration des emprunts}

37 Pour cerner l'intégration linguistique des emprunts, nous avons fait une analyse qui nous a conduit à observer de fortes tendances qui se sont dégagées. Ce sont ces tendances que nous allons discuter dans cette dernière partie de notre réflexion.

Tout d'abord, nous nous sommes rendu compte que si le nom emprunté renvoie aux êtres humains, quelle que soit la syllabe initiale, la classe na- est toujours présente (napadër "prêtre», na-torkaar «troqueur», na-kobaraar «encaisseur», napesaar « peseur »)

39 Ensuite, il a été relevé que si le nom emprunté renvoie à des concepts scientifiques et techniques ou tous les termes modernes, la classe $\mathrm{u}$ - apparaît (u-televison " télévision », u-abiyon "avion », u-mbarku "bateau ») ; (u-nşëment « ciment », umaakna «machine », u-moota "moto », u-fooş «allumette », u-pitaal «hôpital », ulibër « livre », u-ntabël « table ».

40 En mancagne, l'infinitif est une forme nominalisée du verbe qui conserve certaines propriétés typiquement verbales, comme la possibilité d'être accompagnée de tout ou de la partie des arguments du verbe, d'admettre certaines déterminations aspectuelles, modales ou temporelles. Dans cette langue, tous les verbes empruntés sont dans la classe p- qui est la classe qui marque l'infinitif (p-kumsar « entamer », p-judar « jouer », p-markaar «marquer », p-lisaar « repasser»). En analysant les emprunts du mancagne au créole, nous avons remarqué qu'il y a une redondance du morphème de l'infinitif. C'est-à-dire, bien que le verbe emprunté ait la marque de l'infinitif portugais, le locuteur mancagne préfixe toujours le morphème $\mathrm{p}$-.

41 Somme toute, l'intégration des emprunts offre à la fois une homophonie fréquente entre la syllabe initiale et le préfixe de classe. Il suffit d'expliquer que si le mot comporte à l'initiale une séquence susceptible d'être interprétée comme un préfixe de classe, il sera affecté à la classe correspondante.

\section{Conclusion}

L'étude revêt un intérêt particulier car elle nous a permis d'avoir un point de vue global sur l'intégration linguistique des emprunts créoles dans la langue mancagne. Nous avons pu remarquer que c'est une situation, assez intéressante d'un point de vue typologique avec un foisonnement de critères d'intégrations, contrairement à ce qui se fait dans les autres langues du Sénégal. L'intérêt du traitement des emprunts créoles par le système de classification des classes nominales de la langue mancagne réside dans le fait que la quasi-totalité des emprunts sont munis d'un préfixe de classe. En résumé, les mécanismes sont variés et assez complexes, mais nous pouvons retenir que dans cette langue, lorsque la première syllabe du mot emprunté est semblable à un préfixe, celle-ci est réanalysée comme marque de la classe. Dans l'analyse des mécanismes d'intégration, le véritable problème se trouve au niveau des emprunts où le préfixe de classe n'apparait pas au singulier. Dans ce cas de figure ces mots peuvent être analysés comme ayant le préfixe de classe $\Phi$ si dans le système d'accord entre le nom et les différentes unités en rapport avec celui-ci, apparait la marque d'accord correspondante. La situation est plus complexe avec les emprunts qui ne sont pas intégrés morphologiquement car n'ayant ni préfixe de classe au singulier ni au pluriel. 
Dans ce travail, nous avons pu identifier les dix domaines sémantiques les plus prépondérants où apparaissent ces emprunts, dont le plus important est celui de la technologie. Une étude statistique minutieuse va permettre d'établir plus précisément les différents principes qui président au fonctionnement de l'intégration des emprunts et des classes nominales et de rendre compte à la fois de cette homophonie fréquente entre syllabe initiale et préfixe de classe et des autres mécanismes utilisés. Cette étude confirme encore une fois toute la complexité de l'emprunt et des classes nominales.

\section{Abréviation}

43 ACP Acompli

ATR Advanced Tongue Root

C Consonne

CL classe nominale

DEM Démonstratif

DET Déterminant

EX Exemple

INST Instrumental

POSS Possessif

SVO Sujet Verbe Objet

TAM Temps Aspect Mode

V Voyelle

\section{BIBLIOGRAPHIE}

Biasuti, Arturo, Vokabulari Kriol-Portugis Bafatá (Guinée-Bissau). Louvain-Paris : Peeters, 1982.

Biagui, Noel Bernard, Description générale du créole afro-portugais parlé à Ziguinchor, Thèse de doctorat, Université Cheikh Anta Diop, 2012.

Builes, Jean Michel, Manuel de linguistique descriptive, Nathan, 1998.

Demba, Pamanta, « Les emprunts lexicaux peuls au français : Analyse linguistique et sociolinguistique à partir du journal Kabaaru ». Nordic Journal of African Studies 9, 2000, p. 133-151.

Doneux, Jean Léonce, Syllabus du cours de description du manjaku, Document élaboré à l'Université d'Aix-en-Provence, 1993, 35 p.

Dubois, Jean, Giacomo, Mathée, Gespin, Louis, Marcellesi, Christiane, Marcellesi, Jean-Baptiste, Mével, Jean-Pierre, Dictionnaire de linguistique et des sciences du langage, Paris, Larousse, 1973.

Gaved, Tim \& Jon, Stammers, Petit lexique mancagne-français, suivi d'un index français-mancagne, Dakar : SIL Sénégal, 2004, $74 p$.

Karlik, Jan, A Manjako Grammar with Special Reference to the Nominal Group. Ph.D Thesis. London: School of Oriental and African Studies (Department of Phonetics and Linguistics), 1972. 
Kihm, Alain, Aspects d'une syntaxe historique, Études sur le créole portugais de Guinée-Bissau, Thèse de Doctorat de 3e cycle, Paris : Université Paris III, Sorbonne Nouvelle, 1980.

Kihm, Alain, Kriyol syntax: the Portuguese-based créole language of Guinea-Bissau, Amsterdam: Benjamins, 1994.

Kra, Kouakou, « Les emprunts lexicaux du koulango au français et à l'anglais : analyse phonologique ", Cahiers d'études linguistiques, n¹2, 2016, p. 193-2019.

Labatut, Roger, « Les emprunts du peul à l'arabe », Langue arabe et langues africaines, Paris, Conseil international de la langue française, 1983, p41-70.

Loubier, Christiane, De l'usage de l'emprunt linguistique, Montréal : Office québécois de la langue française, 2011.

Mbodj, Chérif, « Esquisse du verbe créole », Annales de la Faculté des Lettres et Sciences Humaines de l'Université Cheick Anta Diop de Dakar, n 17, 1987.

Mohamadou, Aliou, « Fonctionnement morphologique et interprétations sémantiques d'un système classificatoire : l'exemple du peul », Les langues d'Afrique subsaharienne, Paris, éd. OPHRYS, 1998.

Ndao, Dame, L'harmonie vocalique dans les langues du Sénégal, VDM Verlag, 2014.

Ndao, Dame, « Mancanha: a contribution of a culture and language study », Revue du Groupe d'Etudes Linguistiques et Littéraires (G.E.L.L.), n², Université Gaston Berger Saint-Louis, 2018.

Rougé, Jean Louis. Formation et évolution du lexique du créole portugais de Guinée Bissao et de Casamance, Thèse de Doctorat, Lyon : Université Lumière Lyon 2, 1985.

Rougé, Jean Louis, « Uma hipótese sobre a formaçâo do crioulo da Guiné-Bissau e da Casamansa », Soronda 2, 1986, p.28-49.

Rougé, Jean Louis, Petit Dictionnaire Etymologique du Kriol : De Guinée-bissau et Casamance, Bissau INEP, 1988.

Sambieni, Coffi, « Les emprunts français et anglais : une étude de leur intégration phonologique en bali, langue gur orientale », JWAL, 2012, p.83-98.

Trifkovic, Mirjana, Le mancagne : étude phonologique et morphologique, IFAN, Dakar, 1969.

Winford, Donald, An introduction to contact linguistics, Oxford: Blackwell Publishing, 2003.

\section{NOTES}

1. Le Créole n'a rien qui ressemble de près ou de loin aux phénomènes décrits dans d'autres langues comme systèmes de 'classificateurs', 'classes nominales' ou 'genres grammaticaux'. Le terme classe sera employé pour désigner l'ensemble des morphèmes d'un schème d'accord. Il existe plusieurs critères formels pour identifier les classes nominales comme par exemples le préfixe nominal et le schème d'accord.

2. Nous remercions de nouveau Noel Bernard Biagui, Nicolas Quint et Jean François Nunez.

3. Langage, Langues et Cultures d'Afrique Noire.

4. Un grand merci à Pascal Ndeh et Chantal Nzale.

5. Advanced Tongue Root.

6. Le groupe bak appartient à la sous famille « ouest atlantique» de la famille Niger-Congo. On parle de langues «bak » parce que les préfixes de la classe nominale (pluriel des humains), dans ces langues, présente la structure $(b V) k V$ - 
7. En résume dans cette langue, si l'un des deux compléments est un clitique, il apparaît en première position quelle que soit sa fonction (Destinataire ou objet). Si les deux compléments sont des clitiques on a une hiérarchie selon les personnes : $\left(1^{\circ}-2^{\circ}\right.$ pers $>3^{\circ}$ pers $)$. Si le destinataire est une première ou deuxième personne il apparaît premier, sinon, c'est le clitique objet qui apparaît en premier. Pour plus de précisions et d'exemples, se conférer à Ndao (2018).

8. La relativisation d'un circonstant entraîne la disparition de la préposition; et le verbe s'adjoint le suffixe -uy (INST). D'un point de vue synchronique, ce suffixe n'est pas un applicatif étant donné qu'on ne peut pas trouver d'applicatif dans une forme simple indépendante. Toutefois, on peut diachroniquement l'analyser comme un ancien applicatif, dans la mesure où, dans beaucoup de langues, la relativisation de certains circonstants passe nécessairement par leur conversion en objet au moyen d'une dérivation faisant apparaitre le morphème marqueur de l'applicatif.

9. Comme en surface la classe n'est pas visible, peut être aussi il peut s'agir de la classe $\phi$.

\section{RÉSUMÉS}

Le mancagne, langue atlantique parlée au Sénégal et en Guinée-Bissau, est une langue SVO à riche morphologie qui comporte un nombre important d'emprunts. La présente étude est basée sur un corpus recueilli sur le terrain entre 2016 et 2018. L'article analyse les procédés d'appropriation des emprunts lexicaux au créole de Bissau par le mancagne. Il décrit, de ce fait, le processus d'appropriation des emprunts qui tournent essentiellement autour des classes nominales. L'intégration se fait alors selon trois grands procédés : (i) lorsque la première syllabe du mot emprunté est semblable à un préfixe, (ii) quand la syllabe initiale identique à un préfixe de classe est remplacée par un authentique préfixe et (iii) le cas où la syllabe initiale ne correspond à aucune classe.

Mancanha, an atlantic language spoken in Sénégal and Guinea-Bissau, is an SVO language with rich morphology. The current study is based on data collected during fieldwork between 2016 and 2018. This language has a lot of borrowings and this article gives an analysis of the appropriation procedures of lexical borrowing from kriol into mancanha. It describes appropriation processes of mancanha borrowings focusing on nominal classes.

Integration occurs in three main ways: (i) where the first syllable of the borrowed word is similar to a prefix, (ii) where the initial syllable identical to a class prefix is replaced by an authentic prefix and (iii) the case where the initial syllable does not correspond to any class.

\section{INDEX}

Mots-clés : emprunt, mancagne, créole, classe nominale, intégration

Keywords : borrowing, mancanha, kriol, nominal class, integration

\section{AUTEUR}

DAME NDAO

UCAD 